\title{
Evolución del metabolismo de la glucosa sanguínea post-mortem
}

\author{
Sánchez-Alemán E*,, Ramírez-González S**., Rosas-Cabral A. ${ }^{* * *}$
}

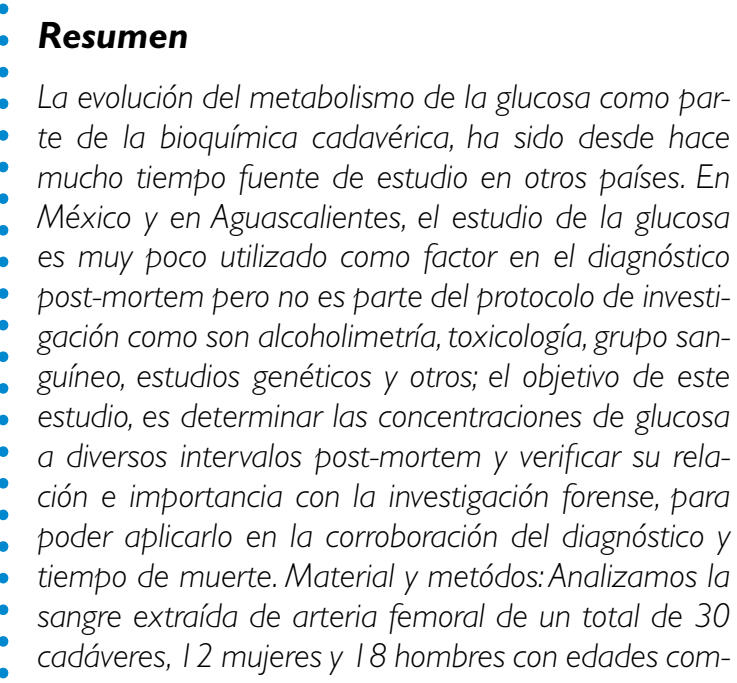

prendidas entre los 22 y 77 años, sin importar la causa de muerte. La determinación de la glucosa se hizo con el apoyo del Instituto Mexicano del Seguro Social, utilizando los aparatos automatizados de química clínica VITROS, mediante la tecnología de química seca. Resultados: El valor de la concentración de glucosa evaluada, representó una constante en evolución, es decir independiente de la edad, causa y horas transcurridas desde el momento de la muerte, se detectaron intervalos regulares de descenso de la glucosa con valores comprendidos entre 6 y $25 \mathrm{mgrl} / \mathrm{l}$ por cada hora, lo que nos permite proponer como una nueva línea de investigación que podría ser herramienta útil para corroborar tiempo de muerte, así como para el diagnóstico del estado hiperglicémico.

Palabras clave: glucosa sanguínea, post-mortem, diagnóstico forense.

\section{Introducción}

Existen numerosos datos de la descomposición del cadáver, sin embargo muchos de ellos se enfocan en aspectos físicos y biológicos que se pueden observar a simple vista o bien detectar mediante el protocolo de autopsia como son livideces, rigidez cadavérica, mancha negra esclerótica, entre otras ${ }^{1,2,3}$. Respecto a la bioquímica cadavérica, existen aun restos de vida, se dan procesos enzimáticos, aunque con algunas diferencias, lo cual no integra a las células para hacer un tejido u órgano funcional. Los objetivos del estudio del comportamiento post-mortem de los compuestos bioquímicos han sido diversos: unos están orientados al establecimiento del momento

* Departamento de Morfología, Centro de Ciencias Básicas, UAA.

** Departamento de Salud Pública, Centro de Ciencias Biomédicas, UAA.

*** Departamento de Medicina, Centro de Ciencias Biomédicas, UAA. 
de la muerte, otros, al mejor conocimiento de la agonía, y otros, al diagnóstico post-mortem ${ }^{4}$. La estimación del intervalo post-mortem (PMI) es uno de los problemas que deben resolverse para el examen forense del cadáver. La estimación precisa de PMI proporciona grandes valores a la investigación penal procesal. Los niveles de los componentes químicos en el humor vítreo humano se cambian con el tiempo después de la muerte, que pueden ayudar a estimar el PMI. Los niveles de ciertos componentes químicos, como el potasio, el magnesio, amoniaco, urea, creatinina, ácido úrico, hipoxantina, ácido láctico y otras, en el humor vítreo humano van a ir aumentando con el tiempo después de la muerte, mientras que otros como el calcio, sodio, enzimas, la glucosa y la vitamina $C$ disminuirán ${ }^{5}$.

La glucólisis es el fenómeno por el cual se disminuye la concentración de glucosa hasta llegar a cero, dado que este se lleva a cabo a nivel celular, este proceso se marca como una constante, ya que los eritrocitos presentes en la sangre son capaces de llevar a cabo este ciclo, sin necesidad de oxígeno. Dado que las enzimas necesarias se encuentran en el plasma sanguíneo, nada impide que este proceso se lleve a cabo en el cadaver ${ }^{6}$. Estudios de glucosa post-mortem se han realizado desde hace tiempo, no solo en sangre, sino en otros líquidos corporales como el líquido cefalorraquídeo y humor vítreo, siendo este último en el cual la glucosa permanecerá constante durante más tiempo debido a la mínima cantidad de células que la consumen, y coincidiendo que la glucosa se degrada rápidamente en sangre e incluso hasta desaparecer en 6 horas el líquido cefaloraquídeo (LCR) Se han hecho estudios comparativos en sangre y humor vítreo analizado la concentración de glucosa con propósitos de un diagnóstico post-mortem, en donde se ha obtenido con éxito, datos que han permitido un diagnóstico de síndrome de muerte de cama por hipoglicemia 7 . Así como también se han reportado casos de detección post-mortem de coma diabético, en donde se hace referencia otra vez al humor vítreo como un fluido sustituto de la sangre en la práctica forense ya que la glucosa sanguínea disminuye rápidamente ${ }^{8}$. Se han reportado casos de cetoacidosis diabética, hipoglucemia e hiperglucemia en diagnóstico post-mortem utilizado también humor vítreo ${ }^{9}, 10$, no solo con el análisis de glucosa, sino también con otros metabolitos como es el betahidroxibutirato asociado con comas diabéticos auxiliando a un diagnóstico post-mortem ${ }^{11}$. La medición del nivel de glucosa en sangre en las cavidades del corazón ha resultado útil para el diagnóstico diferencial de muertes por enfermedad coronaria contra miocardiopatía alcohólica ${ }^{12}$.

El objetivo de este estudio es valorar el metabolismo post-mortem de la glucosa sanguínea, durante las primeras 12 horas después de la muerte, para tomarlo como un auxiliar en el diagnóstico post-mortem relacionado con patologías donde la concentración de la glucosa sea importante para definir la causa de muerte, con ello poder aportar un método adicional en la medicina forense. 


\section{Material y métodos}

Se realizó un estudio observacional, descriptivo, prospectivo, de enero a diciembre 2006, colectando muestras de sangre mediante punción en arteria femoral a intervalos de una hora a un total de 30 cadáveres en el Servicio Médico Forense de la Procuraduría General de Justicia del Estado de Aguascalientes y en el Hospital General de Zona No. 1 del Instituto Mexicano del Seguro Social con diferentes tipos de causas de muerte, siendo 12 del sexo femenino y 18 del sexo masculino, en edades comprendidas desde los 22 a los 77 años.
La evolución de la concentración de la glucosa post-mortem se clasificó con base en el sexo, edad y causa de muerte; se realizaron 3 diferentes tomas de muestra a intervalos regulares de una hora, obteniendo 3 tomas por cadáver, las cuales se analizaron en los aparatos automatizados marca Vitros mediante la tecnología de química seca, utilizando laminillas colorimétricas, que son para una determinación de punto final de mediciones de la glucosa, ácido úrico, colesterol entre otros.

Los datos fueron analizados con el programa GraphPad Prism 3.0.

\section{| | | | | | | | | | | | | | | | | | | | | | | | | | | | | | | | | | | | | | | | | | | | | | | | | | | | | | | | | | | | | | | | | | | | | | | | | | | | | | | | | | | | | | | | | | | | | | | | | | | | | | | | | | | | |}

\section{Resultados}

El total de cadáveres muestreados fueron 30 cuyo sexo, edad y causa de muerte se muestran en la tabla 1 , cabe resaltar que predominaron los hombres (18) con res- pecto a las mujeres (12); también es importante señalar que las enfermedades predominantes fueron infarto al miocardio (6), insuficiencia renal (6) y cáncer (7).

\section{Tabla I}

\section{Comparativo de causa de muerte y género del total de cadáveres muestreados}

GENERO

\begin{tabular}{|c|c|c|c|}
\hline CAUSA de MUERTE & Femenino & Masculino & Total \\
\hline Infarto miocardio & 1 & 5 & 6 \\
\hline Politraumatizado & 1 & 2 & 3 \\
\hline Insuficiencia Renal & 4 & 2 & 6 \\
\hline Cancer & 4 & 3 & 7 \\
\hline Ahorcamiento & & 1 & 1 \\
\hline Traumatismo Craneal & & 1 & 1 \\
\hline Insuficiencia Respiratoria & & 1 & 1 \\
\hline Herida Punzocortante & & 1 & 1 \\
\hline Traumatismo Abdominal & & 1 & 1 \\
\hline Pancreatitis & & 1 & 1 \\
\hline Acidosis Metabólica & 1 & & 1 \\
\hline Neumonía & 1 & & 1 \\
\hline Total & 12 & 18 & 30 \\
\hline
\end{tabular}

Por otra parte y enfocándonos al objetivo de nuestro estudio, en la tabla 2 se muestran los resultados de la concentración de glucosa en cada una de las tomas reali- zadas, así como la diferencia de valores de glucosa entre la primera y la segunda y entre la segunda y la tercera tomas con intervalo de una hora (en negritas). Así mismo, 
se hace notar que los valores basales para cada causa de muerte no fueron iguales y hay mucha diferencia entre ellos, estos resultados eran esperados ya que no son las mismas causales de muerte para cada uno de los cadáveres analizados. Es importante señalar que pretendemos evaluar la variación en la concentración de glucosa de cada una de las muestras y compararla entre los mismos casos con la finalidad de observar si hay un factor constante en esas determinaciones que estén relacionadas con el tiempo de la toma post-mortem, con base en ello, se procedió a hacer el análisis estadístico de las diferencias (negritas) y se presenta en la gráfica 1.

\section{Tabla 2}

Representa los valores promedio de glucosa clasificados en base a la causa de muerte

\begin{tabular}{|lccccc|}
\hline CAUSA de MUERTE & $\begin{array}{c}\text { Glucosa } \\
\text { basal mgr/dl }\end{array}$ & $\begin{array}{c}\text { glucosa } \\
\text { 1a toma }\end{array}$ & Diferencia 1 & $\begin{array}{c}\text { glucosa } \\
\text { 2a toma }\end{array}$ & Diferencia 2 \\
\hline Infarto miocardio & 119 & 104 & 15 & 84 & 20 \\
\hline Politraumatizado & 96 & 75 & 21 & 59 & 16 \\
\hline Insuficiencia Renal & 149 & 130 & 19 & 118 & 12 \\
\hline Cáncer & 116 & 107 & 09 & 81 & 16 \\
\hline Ahorcamiento & 98 & 88 & 10 & 79 & 09 \\
\hline Traumatismo Craneal & 142 & 129 & 13 & 117 & 12 \\
\hline Insuf. Respiratoria & 145 & 129 & 16 & 118 & 11 \\
\hline Herida Punzocortante & 88 & 64 & 24 & 42 & 22 \\
\hline Traumatismo Abdominal & 138 & 128 & 10 & 116 & 12 \\
\hline Pancreatitis & 117 & 106 & 11 & 92 & 14 \\
\hline Acidosis Metabólica & 62 & 51 & 11 & 37 & 14 \\
\hline Neumonía & 103 & 89 & 14 & 64 & 25 \\
\hline Promedio & & & 14.41 & & $\mathbf{1 5 . 2 5}$ \\
\hline
\end{tabular}

Grafica 1. Análisis de los valores de los intervalos entre las tomas: basal, 1 y 2.

Grafica de los valores de los interválos en las tomas de glucosa

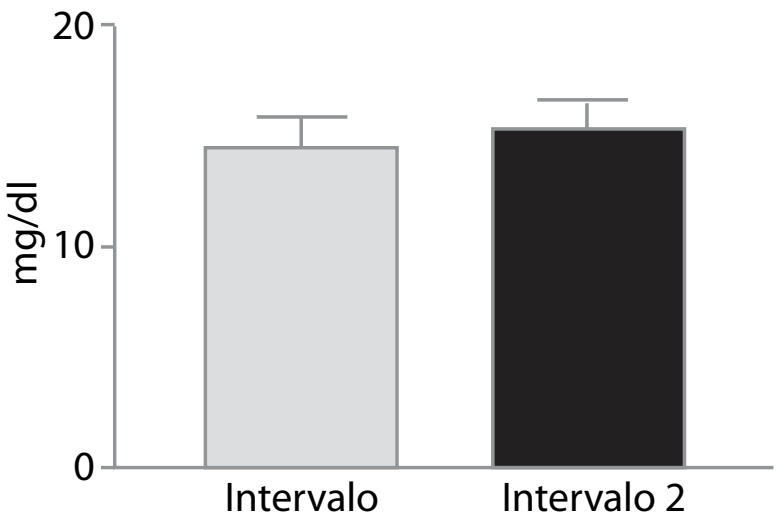

Promedio $14.42 \pm 1.38415 .25 \pm 1.393$

No se observa diferencia estadísticamente significativa entre ellas $(P<0.6755)$. 
Las gráficas siguientes nos hacen referencia de los valores obtenidos de glucosa en base a la edad (gráfica 2), causa de muerte (gráfica 3) y horas transcurridas (gráfica 4). Es importante señalar que no se pretende buscar alguna tendencia para cada una de los parámetros en estudio, sino demostrar que la concentración de la glucosa desciende constantemente a intervalos de tiempo en forma estable y a una velocidad descendente promedio alrededor de $15 \mathrm{mgr} / \mathrm{dl}$ por cada hora.

Hay que resaltar que el espacio entre las líneas que representan la toma basal, a la primera hora y a la segunda hora siempre están separadas en forma constante, lo que significa que independientemente de la edad (Gráfica 2), la glucosa desciende uniformemente.

\section{Gráfica 2}

Representación de la media de concentración de glucosa en $\mathrm{mgr} / \mathrm{dl}$ en base a la edad, para la primera, segunda y tercera toma

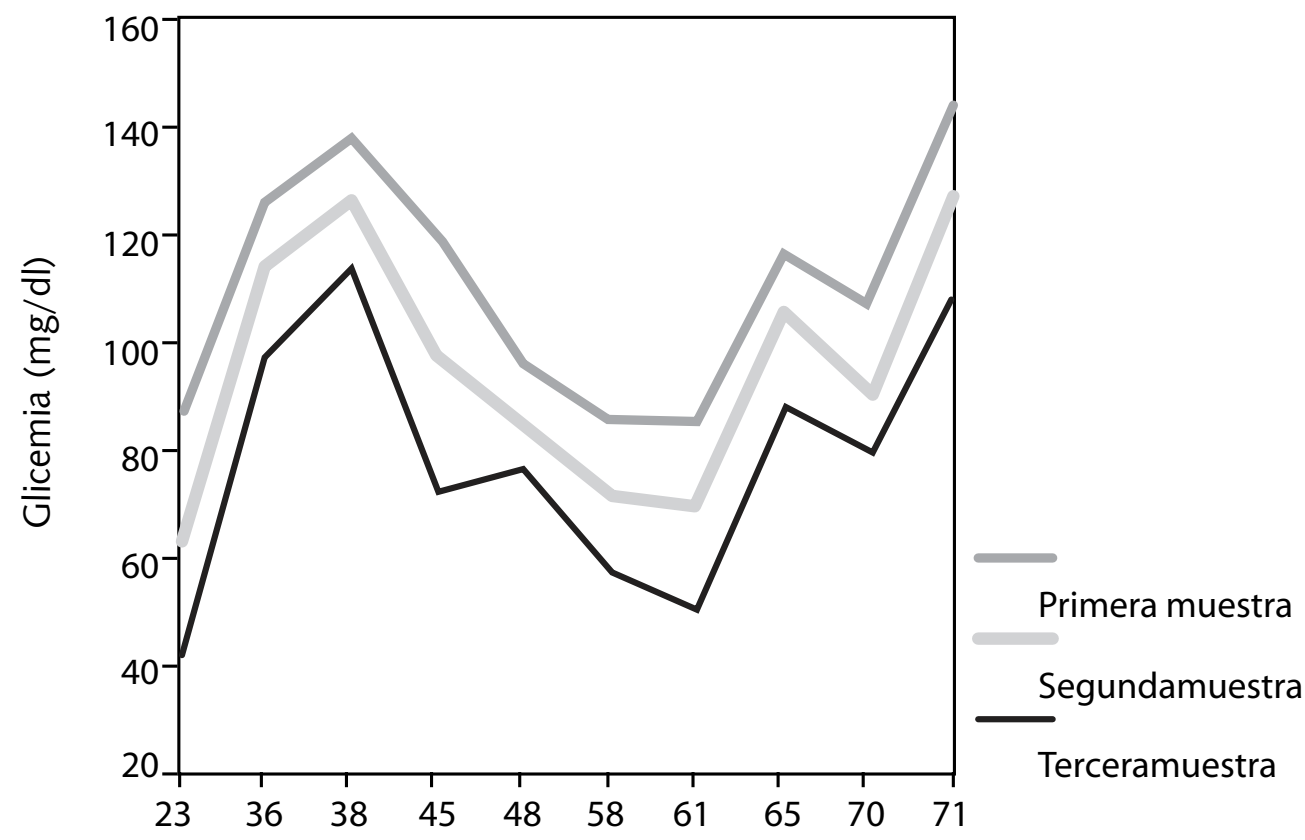

Edad

De igual manera, no hay cambios en la variación de la concentración de glucosa en relación a la causa de muerte de los pacientes analizados (Gráfica 3). 


\section{Gráfica 3}

Representación de la media de concentración de glucosa en mgr/dl clasificados en base a la causa de la muerte, para la primera, segunda y tercera toma.

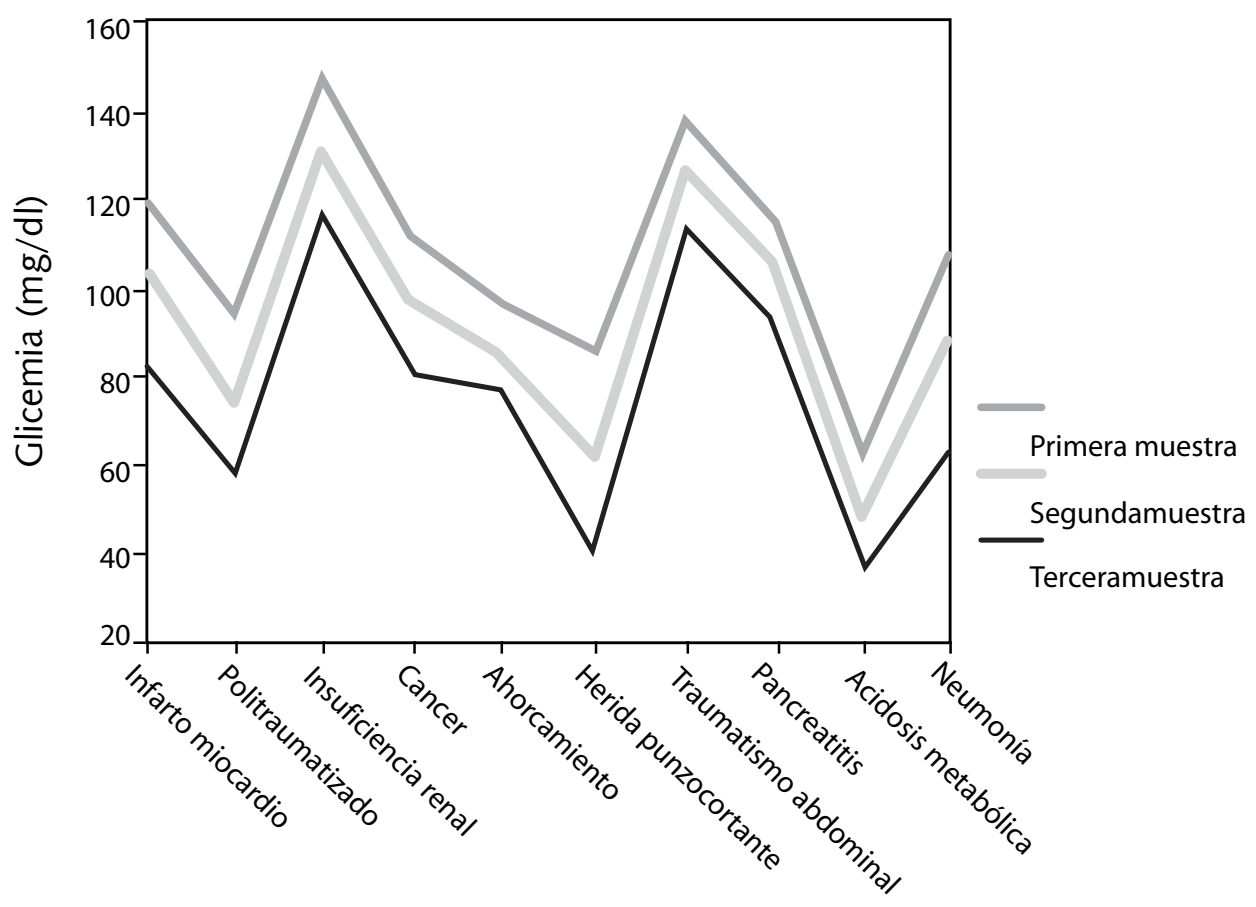

Causas de muerte

La concentración de glucosa sigue descendiendo independientemente de la hora de la toma de la primera muestra.

Grafica 4. Representación de la media de concentración de glucosa en mgr/dl clasificados en base a las horas transcurridas de muerte.

\section{Discusión}

En base al objetivo de este estudio se han revisado los resultados de la medición de la concentración de glucosa en cada una de las tomas post-mortem. Patólogos forenses a menudo dudan en usar los marcadores bioquímicos en sangre, debido al riesgo de los cambios post-mortem y grandes desviaciones respecto de los sujetos sanos. Los análisis bioquímicos de sangre post-mortem, puede ayudar a evaluar el estado patológico y determinar la causa de muerte en el diagnóstico forense, por ejemplo, en la muerte súbita e inesperada, sin causa evidente, o en adultos jóvenes sin causa aparente de muerte ${ }^{13}$. Las esti- 
maciones sugieren que más de 5 millones de ciudadanos de EE.UU. tienen diabetes sin saberlo y están en mayor riesgo de morbilidad y mortalidad, por lo que han evaluado además de la glucosa, la hemoglobina glicosilada como herramientas postmortem para poder identificar a esas personas, obteniendo resultados satisfactorios para llegar al diagnóstico ${ }^{14}$. Los cadáveres muestreados en nuestra investigación tuvieron diferentes causas de muerte, lo cual resulta un dato interesante en cuando a la evolución de la glucosa, ya que mediante estos datos obtenidos se pudo demostrar en cada uno de los casos que la concentración de glucosa disminuyó y pudo detectarse en las todas las tomas realizadas, ya que se hizo el muestreo dentro de las primeras 8 horas post-mortem como lo han realizado otros investigadores ${ }^{15}$. No hay relación entre causa de muerte y edad, ya que se tomaron muestras representativas de muerte natural, homicida y accidental, en donde también se vio afectada de manera independiente, lo mismo respecto a la edad, por lo tanto se puede afirmar que no existe relación con la concentración de glucosa post-mortem, lo cual se corrobora con los valores normales que son iguales en cualquier edad. Sin embargo, se pudo observar un ligero aumento de glucosa en cadáveres pertenecientes al sexo masculino, donde las causas de muerte predominantes fueron el infarto agudo de miocardio, politraumatizados y cáncer. Los que se muestrearon en hospital, pudieron tener aumento de la glucosa basal debido a las características de su enfermedad y probablemente se les trató con solución glucosada, además de otros medicamentos.

Analizando para cada uno de los casos se tiene: Valores de glucosa comprendidos desde $150 \mathrm{mg} / \mathrm{dl}$ en las primeras 2 horas post-mortem y hasta $36 \mathrm{mg} / \mathrm{dl}$ a las $8 \mathrm{hrs}$ después de la muerte.
Los valores de glucosa que se hacen notar en la gráfica número tres, para las causas de muerte resultan ser los más altos para insuficiencia renal y traumatismo abdominal y los más bajos para heridas punzocortantes y acidosis metabólica.

La media de la concentración de glucosa, evaluando su evolución post-mortem representa una constante, es decir independientemente de la edad, causa y horas transcurridas desde el momento de la muerte hasta la primera toma siendo menor a nueve, va disminuyendo en concentración en intervalos regulares con valores comprendidos entre 9 y $25 \mathrm{mgr} / \mathrm{dl}$ por cada hora, con valor promedio de 14.41 para la primera hora y 15.25 para la segunda toma (tabla 2 y gráfica 1), estos son cambios de la glucosa sanguínea post-mortem en toma de arteria femoral, que no han sido reportados por otros investigadores.

La tanatoquimia es una ayuda importante para la determinación de la causa de muerte, aunque tiene sus limitaciones se requiere del conocimiento de distintas circunstancias y datos en el contexto de una determinada situación. Se debe de conocer la bioquímica cadavérica de la glucosa, su metabolismo anabólico en ausencia de oxigeno y así como su relación con diversas enfermedades o causas de muerte para poder aplicar este estudio con éxito, por lo que este trabajo práctico queda abierto a nuevas líneas de investigación, que podrían ser herramientas útiles para corroborar con el tiempo de muerte, así como el diagnostico de diabetes, haciendo grupos de trabajo con occisos con historial de este padecimiento y occisos sin la enfermedad ante mortem, o bien como causa de la muerte como es el choque hiperglicemico. También se podrían formar otros paquetes de investigación clasificando diferentes causas de muerte y su evolución de glucosa sanguínea post-mortem. 


\section{Conclusiones}

Este estudio representa un instrumento de alto valor para el conocimiento de los procesos de descomposición ya que efectivamente la bioquímica cadavérica inicia desde el mismo momento de la muerte, siendo en particular la glucosa que es el carbohidrato principal para la obtención y almacenamiento de energía el que se ve afectado en las primeras 12 horas después del fallecimiento, lo que con una simple medición de glucosa se pueden obtener valiosos datos para un diagnóstico forense mas acertado.

\section{Bibliografía}

1 Vargas, A. E. Medicina Legal. México D.F. 2a Ed. 1999; Editorial Trillas: 73-100.

2 Trujillo N. G. A. Medicina Forense. México D. F. 2002. JGC Editores: 47-54.

3 Martínez Murillo Salvador y Saldivar S. Luis, Medicina Legal, 17a . Edición, México D.F.: Méndez Editores, 2004: 41-71.

4 Gisbert Calabuig Juan Antonio, Medicina Legal y Toxicología, 6a ${ }^{a}$. Edición, Barcelona España: ed. Masson, S.A. 2004: 174-184.

5 Chen YQ, Cai JF, Wen JF. Advances in the studies of post-mortem interval estimation by the levels of chemical components in human vitreous humor after death. Fa Yi Xue Za Zhi. 2009; 25(1):53-6.

6 Murray Robert K., Bioquímica de Harper (traducción de Ma. Del Rosario Carsolio P. Q.F.B.), 14ª . Edición, México, D.F.: ed. El Manual Moderno, 2005: 202224.

7 Tanenberg RJ, Newton CA, Drake AJ. Confirmation of Hypoglycemia in the 'Dead-in-Bed' Syndrome as Captured by a Retrospective Continuous Glucose Monitoring System. Endocr Pract. 2009; 15:1-13.

8 Zilg B, Alkass K, Berg S, Druid H. Postmortem identification of hyperglycemia. Forensic Sci Int. 2009; 185(1-3):89-95.

9 Byard RW, Riches KJ, Kostakis C, Felgate HE. Diabetic ketoacidosis--a possible complicating factor in dea- ths associated with drug overdose: two case reports. Med Sci Law. 2006 Jan; 46(1):81-4.

10 Terekhina NA, Akimov PA. Biochemical analysis of the vitreous body of the eye in post-mortem diagnosis of diabetic coma. Patol Fiziol Eksp Ter. 2005; (2):24-5.

11 Osuna E, Vivero G, Conejero J, Abenza JM, Martínez $P$, Luna A, Pérez-Cárceles MD . Postmortem vitreous humor beta-hydroxybutyrate: it's utility for the postmortem interpretation of diabetes mellitus. Forensic Sci Int. 2005 Oct 29; 153(2-3):189-95. Epub 2004 Nov 6.

12 Astashkina OG, Vlasova NV.The importance of biochemical studies for practical forensic-medical expert examination. Sud Med Ekspert. 2008; 51(4):19-22.

13 Uemura K, Shintani-Ishida K, Saka K, Nakajima M, Ikegaya $\mathrm{H}$, Kikuchi $\mathrm{Y}$, Yoshida K. Biochemical blood markers and sampling sites in forensic autopsy. J forensic Leg Med. 2008; 15(5):312-7.

14 Winecker RE, Hammett-Stabler CA, Chapman JF, Ropero-Miller JD. HbA1c as a postmortem tool to identify glycemic control. J Forensic Sci. 2002; 47(6):1373-9.

15 Khuu HM, Robinson CA, Brissie RM, Konrad RJ. Postmortem diagnosis of unsuspected diabetes mellitus established by determination of decedent's hemoglobin A1c level. J Forensic Sci. 1999; 44(3):643-6. 